\title{
Facilitating Flexible Problem Solving: A Cognitive Load Perspective
}

\author{
Slava Kalyuga • Alexander Renkl • Fred Paas
}

Published online: 30 April 2010

(C) The Author(s) 2010. This article is published with open access at Springerlink.com

\begin{abstract}
The development of flexible, transferable problem-solving skills is an important aim of contemporary educational systems. Since processing limitations of our mind represent a major factor influencing any meaningful learning, the acquisition of flexible problem-solving skills needs to be based on known characteristics of our cognitive architecture in order to be effective and efficient. This paper takes a closer look at the processes involved in the acquisition of flexible problem-solving skills within a cognitive load framework. It concludes that (1) cognitive load theory can benefit from putting more emphasis on generalized knowledge structures; (2) there are tradeoffs between generality and power with respect to specific versus generalized knowledge structures; (3) generalized knowledge structures of "medium" generality are essential for flexible expertise; and (4) cognitive load theory could provide a valuable framework for considering essential attributes of flexible expertise.
\end{abstract}

Keywords Cognitive load · Flexible problem solving · Generalized knowledge structures

Modern society and economy require professionals who can effectively adapt to rapid technological changes (i.e., are flexible or adaptive experts). High rates of technological change require abilities to dynamically adjust cognitive activities based on flexible knowledge, nonroutinely approach new tasks and ideas, and rapidly acquire as well as use new knowledge and skills in practice. The great amount of research effort that has been dedicated to issues of transfer as well as flexibility of knowledge and skills indicates that

S. Kalyuga $(\square)$

School of Education, University of New South Wales, Sydney, NSW 2052, Australia e-mail: s.kalyuga@unsw.edu.au

A. Renkl

University of Freiburg, Freiburg, Germany

e-mail: renkl@psychologie.uni-freiburg.de

F. Paas

Erasmus University Rotterdam, Rotterdam, The Netherlands

e-mail: paas@fsw.eur.nl 
only narrow training for specific routine skills may not be sufficient for developing students' abilities to face new problems in efficient ways (Bransford and Schwartz 1999; Goldstone and Sakamoto 2003; Marton 2006). Hence, an important goal of contemporary education is the development of flexible problem-solving skills as abilities to apply available knowledge in relatively new situations.

Even though much of expert problem solving in familiar domains relies on routine knowledge and skills, efficient flexible performance in new situations may require additional cognitive attributes that allow not only to solve novel problems but also to quickly learn new knowledge if necessary (cf. the concepts of "preparation for future learning" by Bransford and Schwartz 1999, and of "accelerated future learning" by Chi and VanLehn 2007). Examples of such cognitive characteristics may include higher-level cognitive strategies such as self-regulatory performance control strategies and other metacognitive skills (Bransford et al. 1999; Hatano and Inagaki 1986). However, this paper focuses on the role of an organized knowledge base for flexible performance in new situations. It has been recognized that a learner's organized knowledge of interrelationships between essential concepts within a domain is a strong predictor of success in solving transfer problems (Jonassen 2000; Robertson 1990). Such knowledge represents a conceptual framework that could be applied to many different problems within a domain.

In this article, flexible problem-solving skills are considered not as a separate class of skills (for example, generic weak problem-solving methods such as means-ends analysis) but rather as a feature or function of some available domain-specific structures. We argue that these are knowledge structures of relatively higher levels of generality than traditionally considered routine knowledge and skills for dealing with specific problem situations. Also, in this article, similar to most of cognitive load research, the notion of expertise (including flexible expertise) is associated with advanced learners excelling in selected subject areas rather than professional level experts.

Processing limitations of our cognitive system represent a major factor influencing most learning processes, including acquisition of problem-solving skills (Sweller 1988). The acquisition of flexible problem-solving skills also needs to be based on known strengths and limitations of human mind. The essential characteristics of two major components of our cognitive architecture, working memory as an information processor and long-term memory as a store of knowledge, have been well established and become important issues in recent theoretical frameworks for the development of thinking and problem-solving skills (e.g., Demetriou et al. 2002).

Our working memory is severely limited in capacity and duration when dealing with novel information. When solving a novel complex problem, the number of elements of information that need to be processed simultaneously could exceed the available capacity of working memory. For example, we may need to simultaneously keep and process in working memory multiple possible solution paths, compare them, and search and match for additional required information, etc. The ability to manage this cognitive load is essential for successful performance in novel situations. Accordingly, flexible problem-solving skills could be generally considered from a wider perspective as means for managing cognitive (working memory) resources when dealing with novel problem situations. As previously mentioned, this paper focuses on one of such means, that is, organized knowledge structures.

Accumulating organized knowledge structures in specific domains and automating some, usually more basic, procedures represents the most effective means for managing cognitive load when dealing with familiar task domains. These knowledge structures allow us to organize information in working memory by categorizing multiple elements of 
information into higher-level units (Chi et al. 1982). Knowledge-based problem solving in familiar task domains usually proceeds relatively effortlessly by rapidly classifying problem situations and retrieving appropriate strategies and procedures. A well-organized knowledge base in long-term memory is therefore considered as an essential characteristic of effective problem solving in familiar task domains. Would this argument also apply to flexible problem solving in novel situations? And if yes, what types of knowledge would be involved?

\section{Cognitive Load Theory: "Just" a Theory on Acquiring Domain-Specific Routine Expertise?}

According to the evolutionary perspective that has been recently used to underpin major assumptions of cognitive load theory (CLT) (Sweller 2008; Sweller and Sweller 2006), general problem-solving skills such as means-ends analysis and other similar generic problem-solving heuristics belong to a rather vast category of knowledge (called biologically primary knowledge; Geary 2007, 2008) that we have evolved to learn and use mostly implicitly without a need for an explicit instruction. These general problemsolving heuristics are acquired rapidly with minimal effortful learning. Explicit learning and instruction are usually needed for acquiring knowledge and skills that we use in solving problems in specific domains (biologically secondary knowledge according to the above typology). Multiple studies of expertise in different domains, starting from the classical results of De Groot (1965) in chess expertise, emphasized the domain-specific nature of the knowledge structures that experts rely on in their approach to problems rather than generic problem-solving capabilities. In relation to the above dichotomy of domain-specific versus generic biologically primary knowledge, this paper investigates the role of domain-specific knowledge structures of a higher level of generality than the routine low-level knowledge structures directly bound to specific problem situations that are traditionally considered in CLT.

CLT has addressed the issue of flexible problem-solving skills to some extent, however, without considering knowledge structures at a higher level of generality. Two essential means for enhancing learner problem-solving flexibility and transfer capabilities have been traditionally considered in CLT. In the well-structured area of algebra equations, Cooper and Sweller (1987) demonstrated that learners' transfer performance improved notably only after studying a significant number of worked examples paired with problem-solving tasks. Such extensive practice presumably allowed learners to automate basic procedures and devote more working memory resources to making sense of novel aspects of relatively new problem situations. Similar results were obtained by Paas (1992) in the domain of statistics, indicating that solving conventional problems combined with studying worked examples or completing partly worked-out problems was superior to solving conventional problems for attaining transfer. Paas and van Merriënboer (1994) demonstrated that varying problem conditions used in the examples could also enhance learner performance on transfer tasks. Such variability of worked examples would presumably result in more flexible schemas that are applicable to a wider range of specific situations. However, even though increasing the variability of learning tasks and problem types can facilitate the de-contextualization and generalization of procedural knowledge (Barnett and Koslowski 2002; Paas and van Merriënboer 1994), relating many components and levels of knowledge in working memory can, in some cases, also overload working memory, thus decreasing the efficiency of learning. 
CLT assumes that generic strategies such as means-ends analysis and other "weak" problem-solving methods are biologically primary knowledge, and they are considered as not very helpful instructional means, at least not for extending expertise. For example, according to the worked example effect (e.g., Cooper and Sweller 1987), studying examples leads to better skill acquisition than learning by solving problems. This effect is explained by the fact that providing examples prevents learners from applying means-ends analysis, which is not effective for learning, that is, for acquiring schemas for novel tasks (although the specific problem at hand might be solved by this strategy). Hence, CLT has a rather negative attitude toward the role of weak, generic, biologically primary problem-solving methods in instruction for novices. In fact, it was devised in part as a reaction to the failure of such knowledge as instructional means (Sweller 1988). This skeptical view should not extend to instructional benefits of any type of generalized knowledge structures and strategies. Most of such generalized structures are usually developed within specific knowledge domains (i.e., they belong to biologically secondary knowledge) and are applicable within relevant domains only. They occupy medium levels of generality between generic (biologically primary) knowledge and the routine low-level knowledge structures directly associated with specific problem situations (Duncan 2007). In fact, any well-developed field of scientific or technical knowledge reflects a hierarchy of knowledge at different levels of generalization.

There is little doubt that as CLT assumes, relying on low-level domain-specific knowledge structures bound to specific problem situations is the most powerful way to solve corresponding problems and to acquire further knowledge. This well-established finding does not, however, imply that higher-level domain knowledge that is not associated with specific narrow situations is useless. Actually, a number of researchers assume tradeoffs between generality and power of knowledge structures (e.g., Duncan 2007; Friedrich and Mandl 1992; Weinert 1994). For example, while studying general and domain-specific knowledge used by undergraduate students in reasoning about problems in molecular genetics, Duncan (2007) found that students initially formulated their solutions in terms of a general solution frame rather than immediate domainspecific knowledge. This frame outlined and constrained more specific explanations and was filled with more powerful forms of domain-specific knowledge (domain-specific heuristics and domain-specific explanatory schemas). According to Duncan (2007), these components of the solution frame are associated with central mechanisms and entities in the domain and allow students to reason about a variety of both familiar and novel problems. Such solution frames are examples of generalized knowledge structures of medium level of generality that may represent a sensible compromise between generality and power. Especially, they may allow for adapting not yet fully developed expertise to new demands.

It is also interesting to note that some recent studies within the CLT framework also tried to teach skills that are not directly bound to specific problem situations in a domain. For example, Hilbert and Renkl (2009) investigated the acquisition of the learning strategy of concept mapping by example-based learning. Hübner et al. (in press) used worked examples to fostering learning strategies application in reflective writing. Van Merriënboer and Sluijsmans (2009) discussed how CLT could apply to fostering skills of self-directed learning such as assessing task performance or selecting future tasks for learning. In a nutshell, CLT can also provide a useful framework for teaching generalized knowledge and skills. 


\section{A CLT Analysis of Generalized Knowledge Structures: The Case of Function-Process- Structure Schemas}

In order to explore means for enhancing flexibility of problem-solving skills, it is necessary to take a closer look at the nature of the domain-relevant knowledge base. It is possible to distinguish between different levels of such knowledge. At the lower end of the domainspecific level, the knowledge base includes concepts and procedures directly associated with a specific narrow class of problem situations in a domain. Experts rely on this knowledge when dealing with familiar types of specific problems in their area of expertise (Chi et al. 1982; Bransford et al. 1999). If available, such knowledge is used by experts mostly effortlessly without consuming much working memory resources. If it is not available in case of a novel problem, experts may need to resort to search-based weak problem-solving methods. Basic concepts and routine procedures need to be well automated to release cognitive resources for dealing with novel problems. Being an essential condition, such domain-specific knowledge may not be sufficient for successful performance in novel dynamic situations because it is not possible to predict all potential specific situations in advance.

In particular, studying and practicing a large number of specific situations could result in generalization and/or automation of associated knowledge structures. Is such a bottom-up or empirical approach the only way to enhance flexible problem-solving skills? Besides, well-learned or automated domain-specific schemas may not work in new situations. Therefore, another important feature of flexible problem-solving skills is the ability to disengage such narrowly bound schemas in favor of consciously applied higher-level conceptual frameworks and strategies or effortful reasoning and problem solving. Learner abilities to allocate and manage cognitive resources may allow them to withstand high levels of cognitive load involved in such processes. For example, learners may intentionally ignore redundant information that could otherwise cause cognitive overload. One of the assumptions of this contribution is that domain-generalized knowledge structures of medium generality, such as conceptual frameworks or strategic problem-solving approaches in a domain, are useful in this respect.

An example of generalized schematic knowledge developed in technical domains is a framework that includes three related components: functions, processes, and structures (Function-Process-Structure [FPS] schema) (Kalyuga 1984). It is assumed that a technical object (e.g., device or technology) could be characterized by (1) functions (purpose) it was designed for (what is this object for?); (2) natural physical, chemical, or biological processes utilized in the object's operation (how does it operate?); and (3) the object's internal structure including links between its components (what does it consist of?). According to this FPS framework, knowledge of the technical object's operation means knowing why a given set of linked parts performs specific functions utilizing certain natural processes during operation. This triarchic structure effectively defines the nature of technical knowledge as different from purely scientific knowledge. Thus, to understand the operation of the technical object, a learner needs to establish mental connections between these components. There are other similar approaches, for example, the "structurebehaviour-function" framework (Hmelo-Silver and Pfeffer 2004; Gruber and Russell 1996).

Each of the above aspects of technical knowledge may have different levels of generalization: from descriptions of general features to specific details. It is possible to describe an object in very general terms (a global level or general overview) or in more details with different levels of specification. For example, the function of a room air- 
conditioning system could be stated at the most general level as lowering the temperature of a room. At a more detailed level of description, it is taking in warm air, cooling this air down, and then returning this cool air back to the room. At even more detailed level, it is continuously transforming the warm vaporized refrigerant back into a cool liquid form that could be repeatedly used to absorb heat from the air. At the most general level of description, the above major function (lowering the temperature of a room) could be achieved by absorbing heat from the air (the major process). To implement this process, the air-conditioner uses a special substance, a refrigerant (the major part of the structure). Following this most general level, more detailed descriptions the function, structure, and processes of the system could be provided.

When combined together, all aspects, components, and levels of the description create a sophisticated hierarchical knowledge structure. Superficial knowledge about an object may include only some isolated components of this structure. Deep knowledge, in contrast, requires establishing elaborated relations between different components and levels of their description. When constructing complex knowledge of a technical object, processing many elements and interactions simultaneously and making connections among different components may impose a heavy cognitive load. The suggested framework provides a generalized schema that can be used as a deep explanatory principle that may allow effective reasoning about complex systems and account for the relationships across their levels (Goel et al. 1996). The framework also captures the differences between experts' and novices' understanding of the systems. Novices focus on their perceptually available structural components, while for experts, the behavioral (processes) and functional levels serve as the deep principles that organize their knowledge of the system (Hmelo et al. 2000; Hmelo-Silver and Pfeffer 2004).

Instructing for flexible problem solving in a domain could be possibly improved by making higher-level schemas, such as the Function-Process-Structure schema in technical domains, explicit to learners. Acting as deep explanatory principles, such schemas could be flexibly applied in changing situations and transferred across specific areas in the domain (Goldstone and Sakamoto 2003). Such schemas may include knowledge structures at different levels of generality within the domain. All these levels of a schema such as Function-Process-Structure are neither very specific (just applicable only to a specific narrow task class or problem situation within a domain) or absolutely general (confined to all tasks or problem situations in general); they are located on medium level of generality.

In summary, generalized schemas such as FPS can be regarded a useful framework for learning about technical devices on different levels of abstraction. In particular, the structure that such a schema provides can be helpful in dealing with potentially overwhelming cognitive load when trying to understand complex devices.

\section{Function-Process-Structure Schema: Instructional Issues}

Investigating the comparative effectiveness of top-down and bottom-up approaches in acquiring generalized knowledge frameworks such as FPS schemas is an important research task. Two aspects have to be differentiated in this respect. First, when a device can be described on different levels of generality, it can be asked whether instruction should start with the very general level, with the very specifics, or at a medium level. Second, it can be asked whether the FPS schema should be first provided by direct instruction (theoretical approach) or should be induced from exemplars (empirical approach). 
Kalyuga and Hanham (in press) partially investigated the first question related to different levels of generality on which a technical device can be described. The authors suggested that in the technical domain, efficient instruction for flexible problem solving is associated with a top-down approach to learning. Transfer should be supported by first building a general FPS description of a device that can be differentiated later on finer grainsize levels. Schema-based instruction in operation of the air-conditioning system was presented in two alternative formats: (1) hierarchically organized schema-based instruction that proceeded from a very general description to more detailed one, and, finally, to the most specific content (e.g., see the above example of different levels of descriptions for the system's function) and (2) single-level schema-based instruction that provided only the most detailed description of the schematic framework from the beginning. The third, control condition contained conventional single-level nonschema-based instruction that contained otherwise identical information (except explicitly naming different aspects of the schematic description).

During the posttest phase, all 45 participating undergraduate students who were enrolled in a range of subjects not related to the technical areas and were novices in the domain were required to answer the same retention and transfer test questions. Verbal protocols of student performance on transfer tasks were also collected through the audio and videotaping of their actions and verbalizations. Results indicated that the hierarchical schema-based instruction group outperformed the single-level and control groups on transfer posttest problems. The gradual introduction of the supporting schematic framework in a top-down way enhanced transfer performance in this study.

The findings of Kalyuga and Hanham (in press) are in line with the progressive differentiation theory by Ausubel (1960). He proposed a general-to-detail (top-down) instructional sequence according to which general ideas (advance organizers) were presented first, followed by more specific related ideas. The advance organizer-based topdown sequence was considered as effective for transfer outcomes (Mayer 1979). Similarly, the elaboration theory (Reigeluth and Stein 1983) claims that instruction should start from an inclusive picture representing the most general ideas ("the epitome"), and then "zoom in" on each of these ideas providing more details. In the process, it periodically "zooms out" to a wider picture to select the next idea for "zooming in."

The second question previously raised refers to how the schematic frameworks such as FPS should be introduced. For example, should direct instruction in generalized schematic frameworks and principles be presented first followed by further specifications and applications to concrete cases, or should instruction start from specific exemplars followed gradually by different levels of generalizations? Would the optimal sequence depend on levels of learner prior knowledge in a domain?

The argument in favor of the specific-to-general ("empirical") approach could be that developing and practicing task-specific concepts and skills initially to a higher degree of efficiency may free working memory resources for the following construction and elaboration of higher level generalized knowledge structures (cf. Pollock et al. 2002). As learners become more experienced in specific classes of tasks, they may increasingly rely on retrieving and applying available knowledge to handle familiar situations without overloading working memory. This knowledge-based performance would make cognitive resources available for the acquisition of higher-order knowledge and skills.

On the other hand, when high-level conceptual structures ("the big picture") or strategies are directly acquired at the beginning, followed by specific knowledge and practice with procedures (general-to-specific or "theoretical" approach), the general schemas may provide frameworks for dealing with multiple lower-level components, thus also reducing 
the risk of a cognitive overload. It is expected that the general-to-specific approach will outperform the specific-to-general approach for low-knowledge students as it may impose lower levels of cognitive load at initial stages of learning and provide learners with generalized frameworks for managing load at the following stages.

Combining these two approaches (moving from a general structure to its specification and back) could be an effective means for building flexible knowledge and skills. For example, whole-task situations organized into a simple-to-complex sequence of task classes with gradually diminishing levels of instructional support may facilitate the acquisition of generalized schemas for nonrecurrent aspects of complex skills (Four-Component Instructional Design model-4C/ID; Van Merriënboer 1997). Adaptive learning environments that tailor instructional procedures and levels of provided instructional support to changing characteristics of learners (e.g., Kalyuga 2007; Park and Lee 2008) could be especially effective in facilitating flexible problem-solving skills as they may allow balancing the above two approaches in a dynamic and cognitively optimal way for each learner.

\section{CLT as a Framework for Developing Flexible Expertise}

The preceding discussion considered different options for dealing with different levels of knowledge generality as an essential attribute of flexible expertise. Other important attributes of flexible expertise such as well-developed self-regulation and metacognitive skills as well as attitudes also need to be taken into account (e.g., Azevedo 2005; Veenman et al. 2004; White and Frederiksen 2005). A major instructional factor that is related to the above attributes is the level of learner control. Most research in adaptive learning environments in CLT has traditionally dealt with system-controlled environments (e.g., adaptive learning in Kalyuga and Sweller 2004, 2005). However, Salden et al. (2006a, b) concluded that system-controlled adaptive dynamic approaches do not necessarily lead to higher transfer performance than nonadaptive static approaches. Another potential disadvantage of system-controlled instruction is the lack of opportunities for the development of self-regulation skills that are important for flexible expertise.

Therefore, we claim that advanced learners may significantly benefit from learning environments that provide more freedom for students in selecting and structuring their learning tasks or, in the present case, the level of generality that is considered (van Merriënboer et al. 2002). Learner-controlled instruction may not be suitable during the early stages of learning complex skills. Students with low levels of expertise might not use the control appropriately, thus inhibiting their learning (Niemic et al. 1996). However, students could be capable of controlling their learning if they are not cognitively overloaded. Combining system and learner control or varying the level of learner control as expertise and self-regulation skills develop could create the best learning conditions (Van Merriënboer et al. 2006).

Learner-controlled environments could be effective in enhancing learners' metacognitive and self-regulation skills as essential attributes of flexible problem-solving skills. In order to enhance acquisition of such skills, instruction needs to be focused on whole learning tasks rather than on separate fragmented components of complex tasks. Nonroutine components of complex tasks require intentional, conscious, and effortful reasoning as well as transferring strategies and procedures from other task areas. Dealing with unfamiliar task aspects requires schemas for controlled effortful performance, while familiar task aspects rely on automated schemas for effortless performance (Kester et al. 2001). Because of many 
components that need to be processed simultaneously, complex learning tasks may impose a heavy cognitive load. Therefore, it is essential to select appropriate levels of instructional guidance or support for complex task performance that is dynamically tailored to learning situations and cognitive characteristics of learners (van Merriënboer et al. 2003).

With a shared instructional control model, the system selects a subset of tasks from the available task pool based on the current level of learner expertise. This subset is then presented to the learner who makes the final selection (Corbalan et al. 2006). Corbalan et al. (2009) demonstrated that shared control yielded higher transfer performance in the condition of high variability over surface features. In contrast, system control resulted in higher transfer performance in the condition of low variability. Thus, the variability over surface features of the learning tasks may not enhance the flexibility of the learned knowledge and skills by itself, but only in combination with shared control. On the other hand, shared control could be optimized by higher levels of variability over surface features.

According to the recently suggested adaptive guidance strategy (Bell and Kozlowski 2002), learners' progress is constantly assessed, and diagnostic information together with tailored recommendations is provided for making effective learning decisions based on past performance. Available empirical evidence indicates that adaptive guidance improves the acquisition of strategic knowledge and skills and enhances complex transfer skills for advances learners (Bell and Kozlowski 2002).

\section{A Summary in Four Theses}

The basic messages of this contribution will be summarized in four interrelated theses:

\section{CLT could benefit from putting more emphasis on generalized knowledge structures.}

There can be little doubt that low-level domain-specific knowledge structures directly bound to specific problem situations are a necessary prerequisite for both routine and highlevel, flexible expert performance. Nevertheless, generalized domain schemas providing deep explanatory principles that transfer across specific classes of tasks in the domain can be useful when learners are on the way to acquire top-level expertise or when experts have to adapt to novel demands. An example of such a helpful generalized knowledge structure in technical domains is the FPS schema.

2. There is a bandwidth-utility dilemma with respect to specific versus generalized knowledge structures

These are tradeoffs between generality and power of knowledge structures. Without a doubt, specific knowledge is the most powerful way to cope with corresponding problem situations. CLT may also be correct that very general strategies such as generic means-end analysis have very little if any power when complex domain problems have to be solved. Nevertheless, knowledge structures of medium generality have some utility.

3. Generalized knowledge structures of medium generality are essential for flexible expertise.

Knowledge structures of medium generality such as the FPS schema in technical domains are important for flexible expertise as a sensible compromise between generality and power. Especially, they allow for adapting expertise that might also not yet have fully developed to new demands. 
4. CLT is also a valuable framework for considering essential attributes of flexible expertise.

In particular, classical formulations of CLT limit themselves in an unnecessary way when considering just extensive low-level, routine domain-specific knowledge, and systemcontrolled environments as means for helping individuals to cope with demands of intrinsically high cognitive load. From a CLT perspective, knowledge structures of medium generality such as the FPS schema in technical domains can also be regarded as useful in coping with potentially overloading processing demands when working on novel problems in corresponding domains. CLT can also inform about the advantages and disadvantages of different ways to acquire generalized knowledge structures (top-down or bottom-up) and appropriate levels of learner control.

In this contribution, we pleaded for considering the potential value of generalized knowledge structures in CLT research. The future will tell how compelling our plea was by showing whether not only ourselves but also other researchers take up this idea.

Open Access This article is distributed under the terms of the Creative Commons Attribution Noncommercial License which permits any noncommercial use, distribution, and reproduction in any medium, provided the original author(s) and source are credited.

\section{References}

Ausubel, D. P. (1960). The use of advance organizers in the learning and retention of meaningful verbal material. Journal of Educational Psychology, 51, 267-272.

Azevedo, R. (2005). Using hypermedia as a metacognitive tool for enhancing student learning? The role of self-regulated learning. Educational Psychologist, 40, 199-209.

Barnett, S. M., \& Koslowski, B. (2002). Adaptive expertise: Effect of type of experience and the level of theoretical understanding it generates. Thinking and Reasoning, 8, $237-267$.

Bell, B. S., \& Kozlowski, S. W. J. (2002). Adaptive guidance: Enhancing self-regulation, knowledge, and performance in technology-based training. Personnel Psychology, 55, 267-306.

Bransford, J. D., \& Schwartz, D. L. (1999). Rethinking transfer: A simple proposal with multiple implications. In A. Iran-Nejad \& P. D. Pearson (Eds.), Review of research in education (Vol. 24, pp. 61100). Washington, DC: American Educational Research Association.

Bransford, J. D., Brown, A. L., \& Cocking, R. R. (Eds.). (1999). How people learn: Mind, brain, experience, and school. Washington, DC: National Academy Press.

Chi, M., \& VanLehn, K. (2007). Accelerated future learning via explicit instruction of a problem solving strategy. In R. Luckin, K. R. Koedinger, \& J. Greer (Eds.), Proceedings of the 13th International Conference on Artificial Intelligence in Education (pp. 409-416). Amsterdam, Netherlands: IOS Press.

Chi, M., Glaser, R., \& Rees, E. (1982). Expertise in problem solving. In R. Sternberg (Ed.), Advances in the psychology of human intelligence (pp. 7-75). Hillsdale, NJ: Erlbaum.

Cooper, G., \& Sweller, J. (1987). The effects of schema acquisition and rule automation on mathematical problem-solving transfer. Journal of Educational Psychology, 79, 347-362.

Corbalan, G., Kester, L., \& van Merriënboer, J. J. G. (2006). Towards a personalized task selection model with shared instructional control. Instructional Science, 34, 399-422.

Corbalan, G., Kester, L., \& Van Merriënboer, J. J. G. (2009). Combining shared control with variability over surface features: Effects on transfer test performance and task involvement. Computers in Human Behavior, 25, 290-298.

De Groot, A. (1965). Thought and choice in chess. The Hague, Netherlands: Mouton. Original work published 1946. 
Demetriou, A., Christou, C., Spanoudis, G., Platsidou, M., Fischer, K. W., \& Dawson, T. L. (2002). The development of mental processing: Efficiency, working memory, and thinking. Monographs of the Society for Research in Child Development, 67(1), 1-167.

Duncan, R. G. (2007). The role of domain-specific knowledge in generative reasoning about complicated multileveled phenomena. Cognition and Instruction, 25, 271-336.

Friedrich, H.F., Mandl, H. (Eds.) 1992. Lern- und Denkstrategien-ein Problemaufriss [Learning and thinking strategies - a problem definition]. In H.F. Freidrich, H. Mandl (Eds.), Lern- und Denkstrategien. Analyse und Intervention. Hogrefe (D): Göttingen.

Geary, D. (2007). Educating the evolved mind: Conceptual foundations for an evolutionary educational psychology. In J. S. Carlson \& J. R. Levin (Eds.), Psychological perspectives on contemporary educational issues (pp. 1-99). Greenwich, CT: Information Age Publishing.

Geary, D. (2008). An evolutionary informed education science. Educational Psychologist, 43, $179-195$.

Goel, A. K., de Silva, G., Garza, A., Crue, N., Murdock, J. W., Recker, M. M., et al. (1996). Towards designing learning environments. I: Exploring how devices work. In C. Fraisson, G. Gauthier, \& A. Lesgold (Eds.), Intelligent tutoring systems: Lecture notes in computer science. Berlin: Springer-Verlag.

Goldstone, R. L., \& Sakamoto, Y. (2003). The transfer of abstract principles governing complex adaptive systems. Cognitive Psychology, 46, 414-466.

Gruber, T. R., \& Russell, D. M. (1996). Generative design rationale: Beyond the record and replay paradigm. In T. P. Moran \& J. M. Carroll (Eds.), Design rationale: Concepts, techniques, and use. Hillsdale, NJ: Erlbaum.

Hatano, G., \& Inagaki, K. (1986). Two Courses of Expertise. In H. Stevenson, H. Azuma, \& K. Hakuta (Eds.), Child development and education in Japan. New York: Freeman.

Hilbert, T. S., \& Renkl, A. (2009). Learning how to use a computer-based concept-mapping tool: Selfexplaining examples helps. Computers in Human Behavior, 25, 267-274.

Hmelo, C. E., Holton, D., \& Kolodner, J. L. (2000). Designing to learn about complex systems. Journal of the Learning Sciences, 9, 247-298.

Hmelo-Silver, C. E., \& Pfeffer, M. G. (2004). Comparing expert and novice understanding of a complex system from the perspective of structures, behaviors, and functions. Cognitive Science, 28, 127-138.

Hübner, S., Nückles, M., Renkl, A. in press. Writing learning journals: Instructional support to overcome learning-strategy deficits. Learning and Instruction.

Jonassen, D. H. (2000). Toward a design theory of problem solving. Educational Technology Research and Development, 48, 63-85.

Kalyuga, S. (1984). On the content of polytechnic knowledge. Soviet Pedagogics, 6, 44-48 (in Russian).

Kalyuga, S. (2007). Expertise reversal effect and its implications for learner-tailored instruction. Educational Psychology Review, 19, 509-539.

Kalyuga, S., Hanham, J. in press. Instructing in generalized knowledge structures to develop flexible problem solving skills. Computers in Human Behavior.

Kalyuga, S., \& Sweller, J. (2004). Measuring knowledge to optimize cognitive load factors during instruction. Journal of Educational Psychology, 96, 558-568.

Kalyuga, S., \& Sweller, J. (2005). Rapid dynamic assessment of expertise to improve the efficiency of adaptive e-learning. Educational Technology, Research and Development, 53, 83-93.

Kester, L., Kirschner, P. A., van Merrienboer, J. J. G., \& Baumer, A. (2001). Just-in-time information presentation and the acquisition of complex cognitive skills. Computers in Human Behavior, 17, 373-391.

Marton, F. (2006). Sameness and difference in transfer. The Journal of the Learning Sciences, 15, 499-535.

Mayer, R. E. (1979). Can advance organizers influence meaningful learning? Review of Educational Research, 49, 371-383.

Niemec, P., Sikorski, C., \& Walberg, H. (1996). Learner-control effects: A review of reviews and a metaanalysis. Journal of Educational Computing Research, 15, 157-174.

Paas, F. (1992). Training strategies for attaining transfer of problem-solving skill in statistics: A cognitiveload approach. Journal of Educational Psychology, 84, 429-434.

Paas, F., \& van Merriënboer, J. J. G. (1994). Variability of worked examples and transfer of geometrical problem-solving skills: A cognitive-load approach. Journal of Educational Psychology, 86, 122-133.

Park, O., \& Lee, J. (2008). Adaptive instructional systems. In J. M. Spector, D. Merrill, J. van Merrienboer, \& M. Driscoll (Eds.), Handbook of research on educational communications and technology (3rd ed., pp. 469-484). Mahwah, NJ: Erlbaum Associates.

Pollock, E., Chandler, P., \& Sweller, J. (2002). Assimilating complex information. Learning and Instruction, 12, 61-86.

Reigeluth, C. M., \& Stein, F. S. (1983). The elaboration theory of instruction. In C. M. Reigeluth (Ed.), Instructional design theories and models: An overview of their current status. Hillsdale, NJ: Lawrence Erlbaum. 
Robertson, W. C. (1990). Detection of cognitive structure with protocol data: Predicting performance on physics transfer problems. Cognitive Science, 14, 253-280.

Salden, R. J. C. M., Paas, F., van der Pal, J., \& van Merriënboer, J. J. G. (2006a). Dynamic task selection in a flight management system training. The International Journal of Aviation Psychology, 16, 157-174.

Salden, R. J. C. M., Paas, F., \& van Merriënboer, J. J. G. (2006b). A comparison of approaches to learning task selection in the training of complex cognitive skills. Computers in Human Behavior, 22, 321-333.

Sweller, J. (1988). Cognitive load during problem solving: Effects on learning. Cognitive Science, 12, $257-285$.

Sweller, J. (2008). Instructional implications of David Geary's evolutionary educational psychology. Educational Psychologist, 43, 214-216.

Sweller, J., \& Sweller, S. (2006). Natural information processing systems. Evolutionary Psychology, 4, 434-458.

Van Merriënboer, J. J. G. (1997). Training complex cognitive skills: A four-component instructional design model for technical training. Englewood Cliffs, NJ: Educational Technology Publications.

Van Merriënboer, J. J. G., \& Sluijsmans, D. M. A. (2009). Toward a synthesis of cognitive load theory, fourcomponent instructional design, and self-directed learning. Educational Psychology Review, 21, 55-66.

Van Merriënboer, J. J. G., Schuurman, J. G., de Croock, M. B. M., \& Paas, F. (2002). Redirecting learners' attention during training: Effects on cognitive load, transfer test performance, and training efficiency. Learning and Instruction, 12, 11-37.

Van Merrienboer, J. J. G., Kirschner, P. A., \& Kester, L. (2003). Taking the load off a learner's mind: Instructional design principles for complex learning. Educational Psychologist, 38, 5-13.

Van Merriënboer, J. J. G., Sluijsmans, D., Corbalan, G., Kalyuga, S., Paas, F., \& Tattersall, C. (2006). Performance assessment and learning task selection in environments for complex learning. In D. Clark \& J. Elen (Eds.), Advances in learning and instruction (pp. 201-220). Amsterdam: Elsevier Science.

Veenman, M. V. J., Wilhelm, P., \& Beishuizen, J. J. (2004). The relations between intellectual and metacognitive skills from a developmental perspective. Learning and Instruction, 14, 89-109.

Weinert, F. E. (1994). Lernen lernen und das eigene Lernen verstehen [Learning to learn and the understanding own learning]. In K. Reusser \& M. Reusser-Weyeneth (Eds.), Verstehen. Psychologischer Prozeß und didaktische Aufgabe (pp. 183-205). Bern: Huber.

White, B., \& Frederiksen, J. (2005). A theoretical framework and approach for fostering metacognitive development. Educational Psychologist, 40, 211-223. 